\title{
Biosurfactants - Nature's Solution for Today's Cleaning Challenges
}

\author{
Jakob J. Mueller* and Hans H. Wenk
}

\begin{abstract}
Biosurfactants are surface-active molecules, developed by nature through evolution and naturally produced by different microorganisms. The most prominent examples are rhamnolipids and sophorolipids, molecules which contain hydrophilic sugar head groups and hydrophobic alkyl residues leading to an amphiphilic behavior with unique properties. Recent developments in the field of biotechnology enable the large-scale production of these biological molecules. The raw material basis is $100 \%$ renewable since sugars and oils are used as major raw materials. Additionally, biosurfactants are fully biodegradable, which allows the path back into the natural cycles. In comparison to established standard surfactants like SLES/SLS (sodium laureth (ether) sulfates) or betaines, rhamnolipids are much milder and, at the same time, show similar or even better performance in household or personal care applications. Foam behavior, solubilization and cleaning effectiveness are examples where these natural substances give excellent results compared to the synthetic benchmarks. The commercialization of biosurfactants at industrial scale now offers alternatives to consumers seeking sustainable solutions, without compromising performance. Biosurfactants combine both and set a new standard for surfactant applications.
\end{abstract}

Keywords: Biodegradable $\cdot$ Biosurfactants $\cdot$ Renewable $\cdot$ Rhamnolipids $\cdot$ Sophorolipids

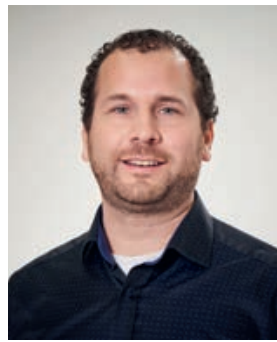

Dr. Jakob Mueller has a Diploma and a PhD in Bioprocess Engineering from Hamburg University of Technology (TUHH). In 10 years with Evonik Operations $\mathrm{GmbH}$, he has worked in several positions in R\&D, technology development and production support in the field of biotechnology, e.g. large-scale production of bio-amino-acids. $\mathrm{He}$ is now responsible for the coordination of $R \& D$ activities for biosurfactants and new technologies.

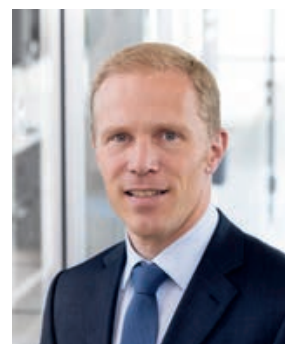

Dr. Hans Henning Wenk has a $\mathrm{PhD}$ in Chemistry from Bochum University. He joined Degussa (now Evonik) in 2002 and has held numerous positions in R\&D and Innovation Management for Personal Care, Home Care, and Food Ingredients. From 2010 to 2013, he was a member of the board of SEPAWA e.V., the German association of the cosmetic and detergent experts. In 2019, he assumed his current position as Global Head of R\&D for the business line Care Solutions of Evonik.

\section{Introduction}

Biosurfactants are amphiphilic molecules naturally synthetized by biological organisms like microorganisms, plants, and animals. ${ }^{[1]}$ Usually, these substances contain at least a hydrophilic head, and a lipophilic tail group. The combination of both groups and their specific hydrophilic-lipophilic balance defines their unique physical properties. Due to their amphiphilic nature, biosurfactants move and act on phase boundaries, leading to changes of surface tension, having effects on foam behavior, or improving the solubilization of oils and fats in aqueous solutions. ${ }^{[2]}$ All these features play an important role in numerous personal and household care applications, products used by all of us in our daily life. One basic characteristic of biosurfactants is their natural origin. Microorganisms like Pseudomonas aeruginosa can produce rhamnolipids, ${ }^{[3,4]}$ a type of molecule consisting of one or two rhamnose units and one or two hydroxy fatty acids with different alkyl chain length (Fig. 1). For the biosynthesis, the organism needs only raw materials like sugars, oils and fats and the product can be considered $100 \%$ biological - an important criterion in consumer perception. Pushed by the current discussion about climate change, the increasing awareness about our own health and sustainable production of food and consumer products, there is a rapidly increasing demand for renewable, natural, and sustainable ingredients.

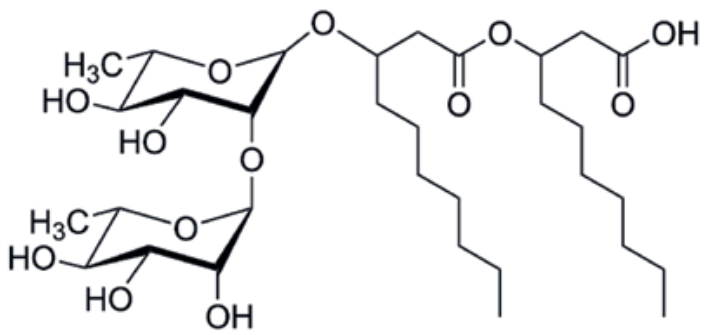

Fig. 1. Chemical structure of a di-rhamnolipid molecule. It consists of two rhamnose units, linked via an ether bond with two hydroxy fatty acid residues. Usually, the chain length of the hydroxy fatty acids is between $\mathrm{C} 8$ and $\mathrm{C} 12$. The final acid function gives the molecule an ionic behavior with a $\mathrm{pH}$ dependency.

For the past century, the cleansing properties of most household cleaning and cosmetic products have been based on surfactants derived from petrochemical sources. In response to shifting consumer demand towards sustainable products, this fast-growing $\$ 40$ billion global market for surfactants is now undergoing a rapid transition towards green surfactants that are fully biodegrad- 
able, minimize $\mathrm{CO}_{2}$ emissions and are not harmful to aquatic or other natural organisms. ${ }^{[5]}$

However, early generations of bio-based surfactants developed since 2000 have faced several challenges relating to raw material sourcing and manufacturing that constrict their green credentials. Most of all, there have been few, if any, bio-based surfactants that have been able to generate the cleaning and foam-generating functional properties of traditional raw materials. These unmet market needs are addressed by several players in the chemical industry, however only a few have commercialized the products with respective amounts, necessary quality, and functionality. ${ }^{[6]}$ Multi-ton quantities of sophorolipids and rhamnolipids are already available today and used for example in different hand dishwash and cosmetic applications (Fig. 2). A world-scale-plant to produce rhamnolipids is currently in the planning and implementation phase. ${ }^{[7]}$

\section{Technology}

Nature developed biosurfactants by evolution: they play a specific role for the organisms synthetizing them. ${ }^{[8]}$ This function in nature does not necessarily follow the logic of an industrial process, since the focus and intentions are different. Therefore, usually the organisms need to be optimized to provide higher yields and form fewer unwanted by-products, which is done by wellknown approaches like directed evolution, ${ }^{[9]}$ random mutagenesis, rational approaches and specific gene transfers, e.g. by new technologies like CRISPR/Cas. ${ }^{[10]}$ The target of the production process is to achieve a certain product quality with an adequate technical effort, directly correlated to the production costs. For the industrial production of the rhamnolipid product, a biotechnological process is used, which consists of a fermentation, followed by a downstream process (Fig. 3). In the fermentation step the microorganisms are converting sugars into the target product. The sugar is usually obtained from corn, which is converted to dextrose syrups by wet milling or dry milling processes. The utilization of sugars from plant residues or other waste streams is under investigation for biosurfactant production ${ }^{[11,12]}$ with further potential to improve the overall sustainability profile. ${ }^{[13]}$ As the production host, Pseudomonas putida, a non-pathogenic organism, is used. The product, a mixture of different rhamnolipids, is excreted to the liquid phase of the fermentation broth. Reaching high titers in this process is challenging, since the product triggers the formation of foam, which becomes even more challenging in aerated bioreactors, the industrial standard for aerobic fermentation processes. There are several approaches to deal with this issue including using mechanical foam breakers, antifoam addition or adjusting the process conditions like pressure and aeration. After the fermentation phase the cells are inactivated and removed from the fermentation broth. This byproduct can be treated in anaerobic wastewater treatment processes for biodegradation and biogas formation. In the following downstream process, the rhamnolipids are separated and purified from the fermentation broth to a high concentrated and highly pure aqueous solution.

\section{Specific Properties of Biosurfactants}

Biosurfactants are produced by renewable raw materials, like sugars or oils and fats. These basic raw materials make them $100 \%$ renewable, which is a strong argument in times where customers are demanding more sustainable products. ${ }^{[14]}$ Additionally, no tropical oils are used, unlike in the group of biobased surfactants. But a sustainable product alone is not enough, the performance and economics of biosurfactants needs to be at least a similar level compared to chemical equivalents. In this case, biosurfactants must compete with established molecules like sodium laureth ether sulphate (SLES, SLS) or betaines. In this section, three examples are presented showing the unique properties and strong performance of rhamnolipids.

\subsection{Aquatic Toxicity and Biodegradability}

For every product produced today, it is important that its substances can be degraded and fed back to natural cycles. Additionally, the amphiphilic behavior can have certain toxic effects on living organisms. Rhamnolipids, due to their natural background, show a significantly lower aquatic toxicity and a maximum biodegradability compared to other standard and widely used surfactants. To test the acute effects on water organisms, the water organisms Daphnia magna (small crustacean, common name water flea) and green algae Pseudokirchneriella subcapitata were selected. For both organisms, the EC50 (half maximal effective concentration) and No Observed Effect Concentration (NOEC) values were determined, which are standard tests for the determination of ecological toxicity. For the organism Daphnia magna, no increased mortality up to a concentration of $94.6 \mathrm{mg}$ rhamnolipid per liter after 48 hours was detected (acute NOEC value). The EC50 value
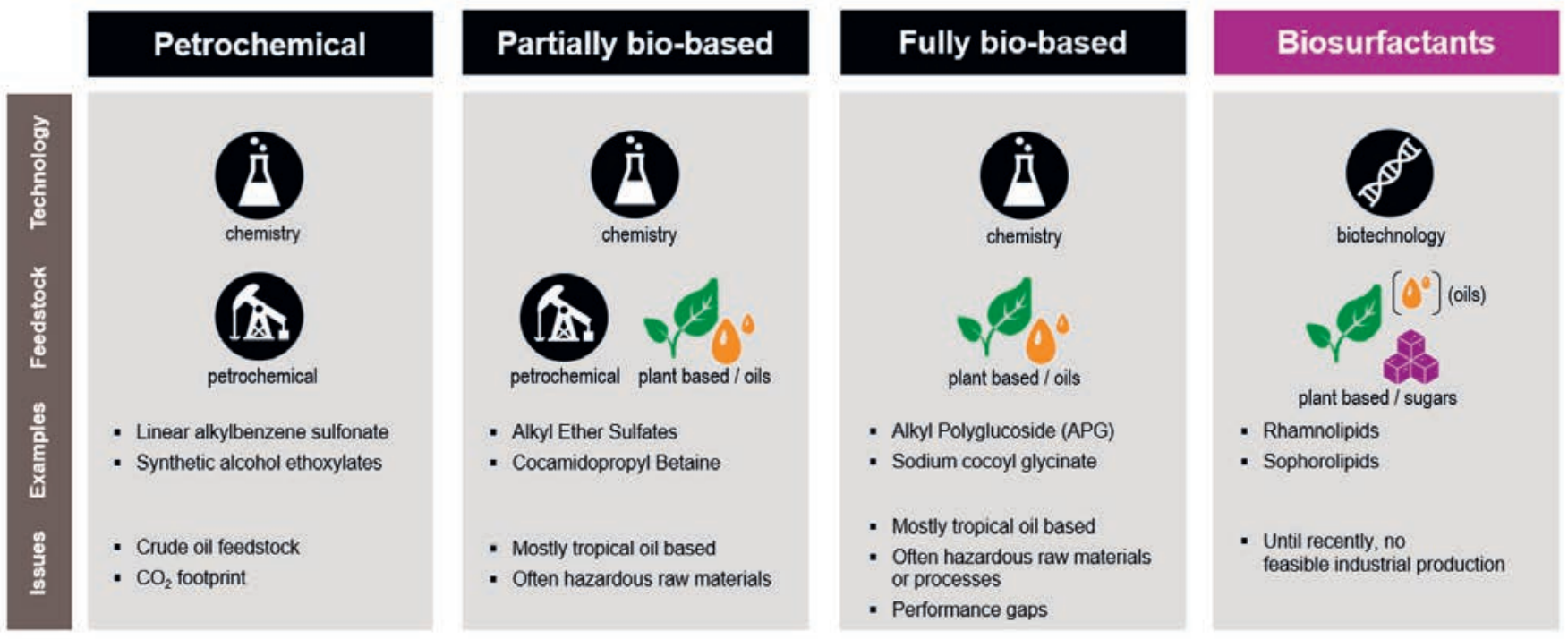

Fig. 2. Why are biosurfactants unique? Biosurfactants are based on renewable resources and the production technology is based on a biotechnological process. The challenges with large-scale production were overcome recently and all signs point in the direction of a significant increase in market share in the coming years. 


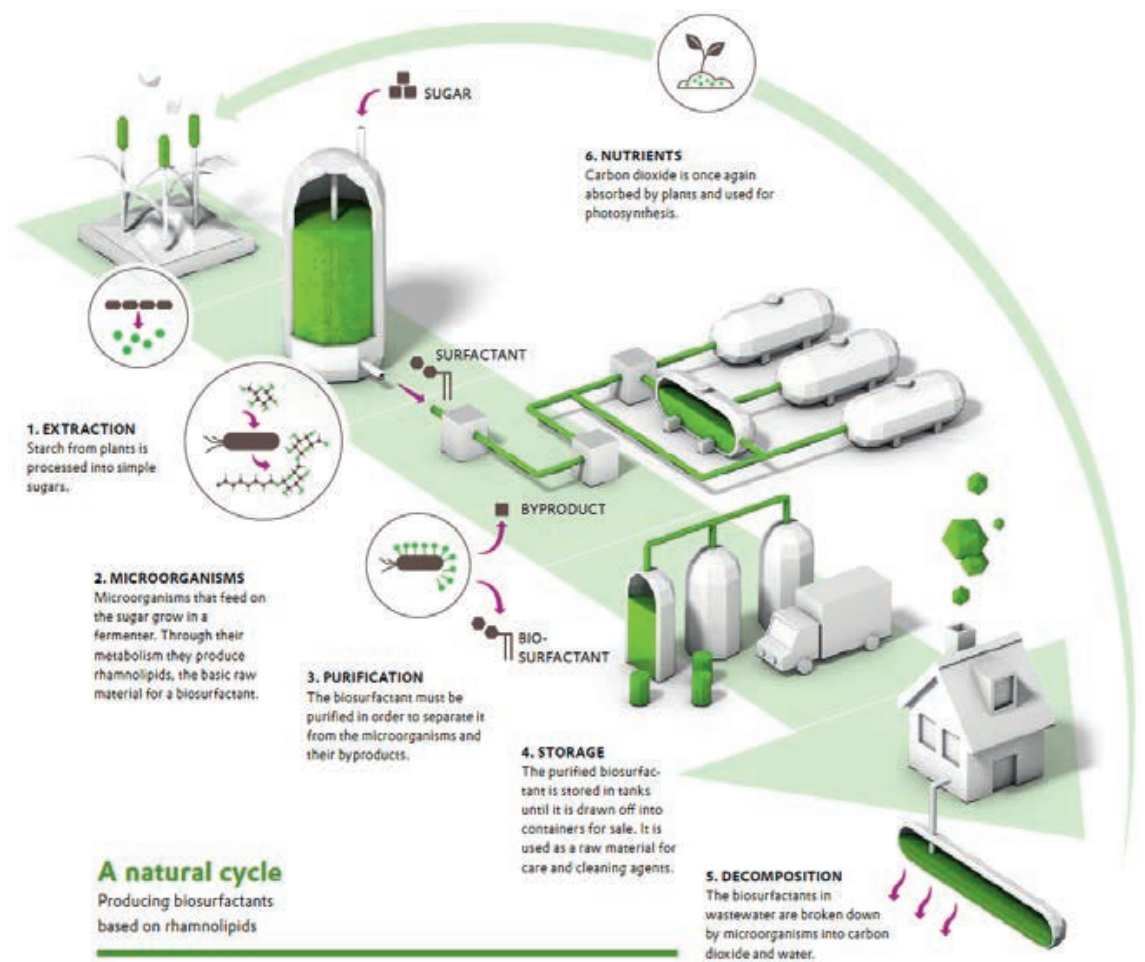

Fig. 3. Process overview. To produce rhamnolipids, natural sugars are used. These are extracted from plant material (e.g. starch from corn) (1). In the fermentation step, the sugar is converted to the product (2). The product is purified in a dedicated downstream process (3). After storage and application, the product, due to its biological degradability, is fed back to the natural cycles (4 and 5). was estimated to be $>196 \mathrm{mg}$ rhamnolipids per liter. For the green algae Pseudokirchneriella subcapitata, the acute toxicity was determined within 72 hours. No growth or yield inhibition was detected for the product concentrations used. Therefore, the NOEC value was calculated to be $>220 \mathrm{mg}$ rhamnolipid product per liter (based on an $84 \%$ pure rhamnolipid product). The EC50 value is based on the same value but is referred to as a $100 \%$ rhamnolipid product. This led to an EC50 value of $>185 \mathrm{mg}$ rhamnolipid per liter. Comparing these numbers with the ecological toxicity results for the surfactants SLES 1EO (sodium laureth ether sulfate), alkyl poly glycosides (APG) C10-16, cocoamidopropyl betaine, sodium cocoyl glycinate and disodium cocoyl glutamate, differences are observed (Fig. 4). The surfactants shown have EC50 values in the range of approximately $2-60 \mathrm{mg} / \mathrm{L}$ and NOEC values between approximately 1-22 $\mathrm{mg} / \mathrm{L}$. Rhamnolipids indicate significantly better EC50 and NOEC values for both organisms compared to the above-mentioned standard surfactants. These results confirm that rhamnolipids have a significantly lower impact on aquatic organisms and show better bio-compatibility. At the same time, rhamnolipids are $100 \%$ biodegradable either via aerobic or anaerobic degradation. Those aspects make rhamnolipids the perfect solution for many applications. Using materials with a biological origin and simple biodegradability is important for products which are rinsed off, like shampoos and washing solutions. Rhamnolipids have valuable advantages for the aspects presented here and outperform well-established products.

\subsection{Mildness}

The cleaning behavior, solubilization, and foaming behavior are typical and important performance indicators of surfactants.

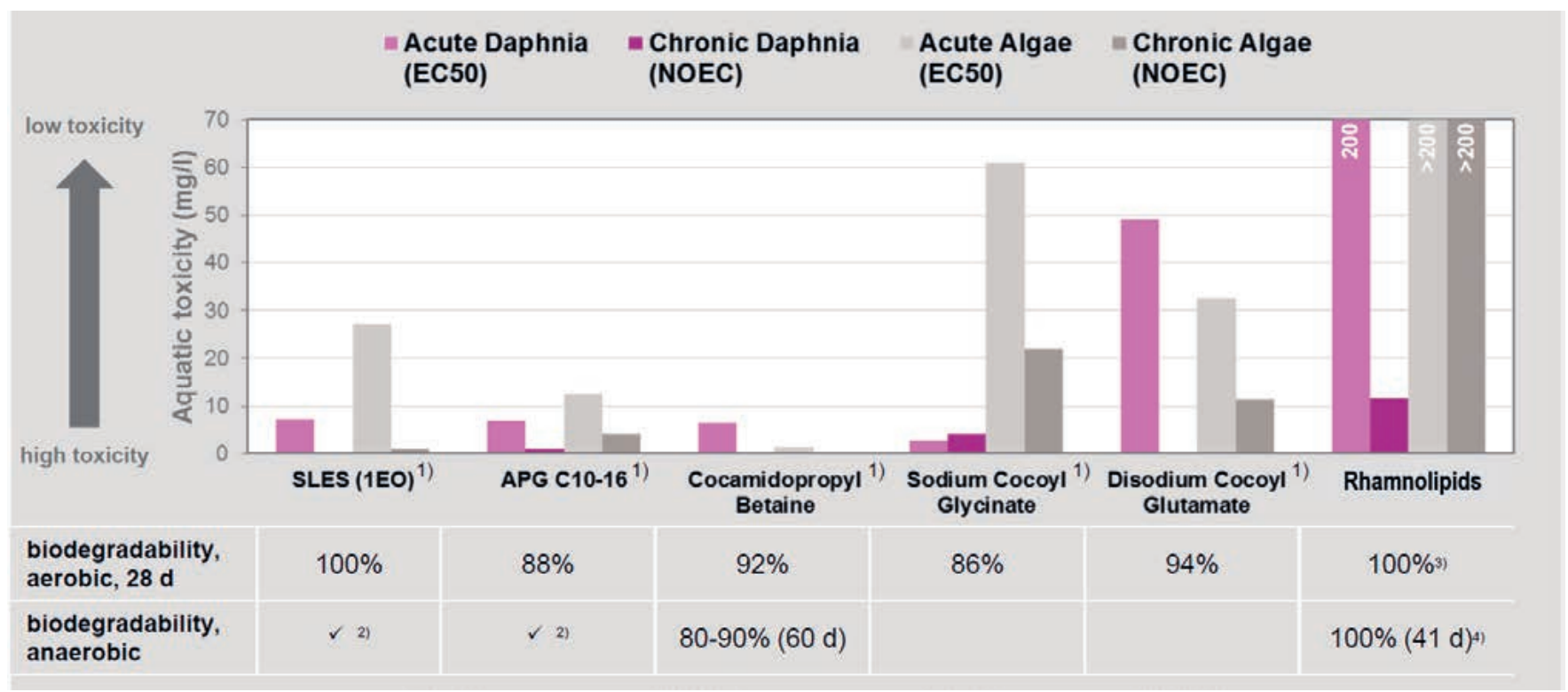

Fig. 4. Comparison of aquatic toxicity and biodegradability for different industrial relevant surfactants. Rhamnolipids show the lowest aquatic toxicity and are $100 \%$ biodegradable. 
In cases where the surfactant meets human skin, eyes and mucosa, the product mildness also plays an important role. In an ideal world, the surfactant performance and mildness should go hand in hand and not be compromised. The interaction of biosurfactants with proteins, known as sensitive molecules with regard to temperature or $\mathrm{pH}$, is a common indicator for surfactant mildness. It can be measured by the red blood cell or Zein test, where denaturation effects on specific proteins are analyzed. Rhamnolipids show a significantly higher L/D value (lysis-to-denaturation ratio) in the red blood cell test compared to standard surfactants (Fig. 5), which is an indication of significant lower protein denaturation. The same result was obtained when the surfactants were added to a suspension containing the maize protein Zein. A reduction of turbidity is a sign of denaturation, since the denaturated Zein has a better water solubility compared to the native form. Rhamnolipids show reduced interaction with the chemical structure of proteins compared to standard surfactants, here shown for sodium laureth ether sulfate and cocoamidopropyl betaine (Fig. 5). These results demonstrate the superior behavior of rhamnolipids in terms of mildness and low protein interaction, which is reasonable, since due to their natural origin from microorganisms, a negative effect on enzymes and proteins would be a disadvantage in evolution.
This property is a significant advantage for applications like shampoos, hand soaps, but also manual dishwashing detergents, where the human skin is in direct contact with the surfactant.

\subsection{Foam Behavior}

Formation of foam is an important performance parameter for cleaning solutions and specific cosmetic applications. Consumers prefer products with good and long-lasting foam formation in the field of hand dishwashing, shampoos and shower/bath products. Foam supports the sensory sense of cosmetic and cleaning products and is a strong indicator for a good quality cleaning performance. The foam structure and stability are usually analyzed to compare the performance between different products. In the Dynamic Foam Analyzer (DFA) method (Fig. 6), it has been confirmed that rhamnolipids give a dense and stable foam, which is comparable to industrial-standard products like sodium laureth ether sulphate (SLES) and cocoamidopropyl betaine (CAPB). Also, for the foam kinetics, which can be analyzed by a SITA foam tester (SITA lab technologies), the required energy input for getting to a high volume in rhamnolipids is lower than for standard surfactants (Fig. 6). That makes the foam formation more efficient and quicker.

\section{Red Blood Cell test results}

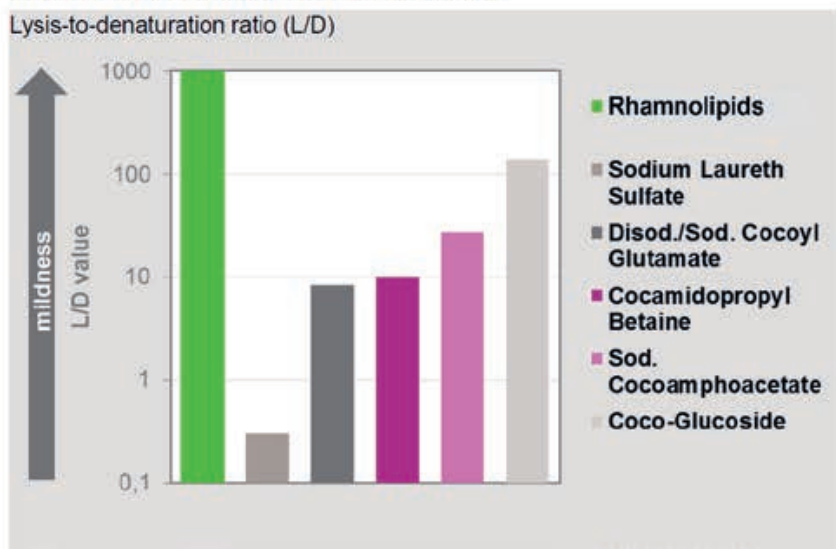

\section{Zein test results}

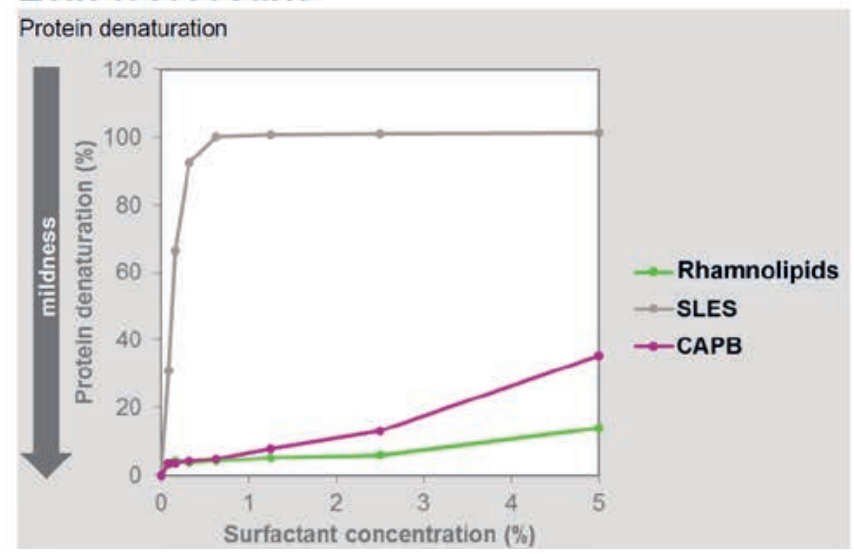

Fig. 5. Mildness comparison for different surfactants. The mildness was measured by the red blood cell and the Zein tests, both tests indicate the protein denaturation impact. Rhamnolipids show significantly reduced protein denaturation compared with different standard surfactants in both tests. CAPB = Cocoamidopropyl Betaine; SLES = Sodium Laureth Sulfate; \% = \% active matter.

\section{Foam structure}

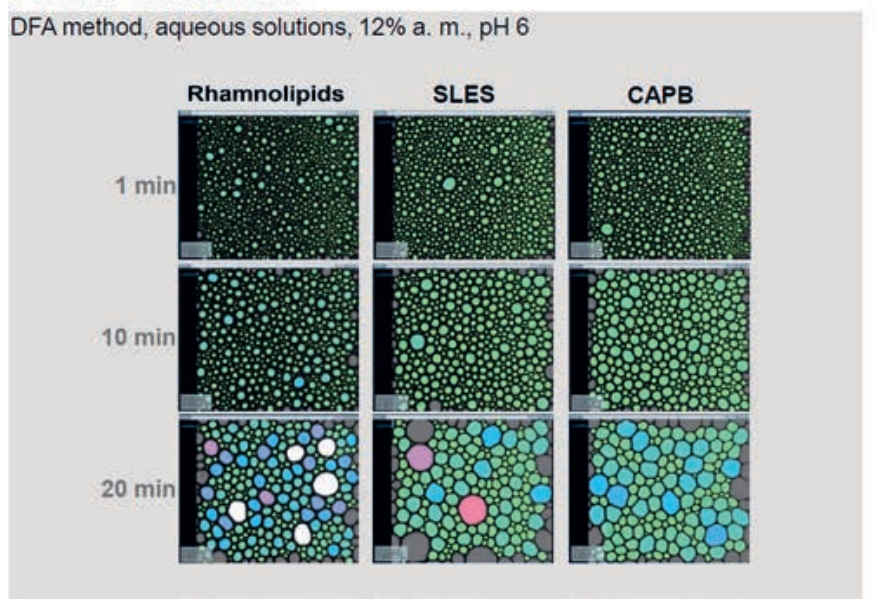

\section{Foam kinetics}

SITA method, $0.5 \%$ a. $\mathrm{m}$., $\mathrm{T}=30^{\circ} \mathrm{C}$, Water $\sim 10^{\circ} \mathrm{dH}, 1500 \mathrm{rpm}, \mathrm{pH} 6$

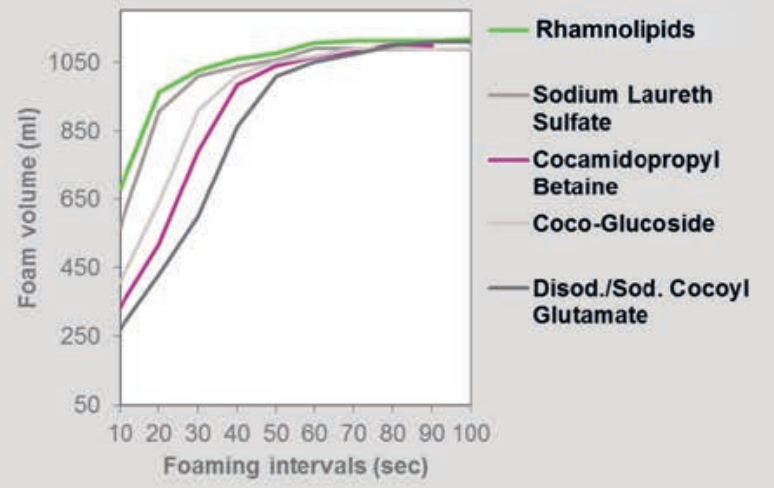

Fig. 6. Foam structure and foam kinetics of rhamnolipids with different standard surfactants like SLES (sodium laureth ether sulphate), CAPB (cocoamidopropyl betaine) and other. The foam forming kinetic is faster and the foam structure is denser, both indicators for a good cleaning and application performance. 


\section{Summary and Conclusion}

Industrially produced biosurfactants are a class of surfactants based on renewable resources and green and biological production technologies that have become newly commercially available. Recent breakthroughs in technology enable the large-scale production of biosurfactants like rhamnolipids and sophorolipids. In contrast to examples where compromises must be made when changing to alternative green and renewable products, rhamnolipids offer excellent technical, sensory and sustainability performance. Additionally, rhamnolipids show significant advantages in the field of mildness, biodegradability and lower eco-toxicity. This makes this product much more compatible with nature and enables it to enter the natural recycling streams. The strong customer demand for clean and renewable solutions, especially in cosmetics and cleaning applications, led to a significant increase of market growth for biosurfactants. The availability of commercial production processes is a major game changer in supporting this development. We are at the beginning of a new era for renewable, green and effective surfactant solutions with an expanding product portfolio, inspired by nature.

Received: May 14, 2021

[1] G. Soberón-Chávez, R. M. Maier, in 'Biosurfactants: A General Overview', in 'Biosurfactants. Microbiology Monographs', vol 20., Eds. G. SoberónChávez, Springer, Berlin, Heidelberg, 2011, p. 1-11.

[2] K. Brandt, V. Dahl, A. Muss, S. Blaschek, Z. Drljaca, S. Volmer, H. H. Wenk, SOFW J. 2018, 144, 15.

[3] G. Soberón-Chávez, F. Lépine, E. Déziel, Appl. Microbiol. Biotechnol. 2005, 68, 718, https://doi.org/10.1007/s00253-005-0150-3.

[4] P. Eslami, H. Hajfarajollah, S. Bazsefidpar, RSC Adv. 2020, 10, 34014, https://doi.org/10.1039/D0RA04953K
[5] R. Jahan, A. M. Bodratti, M. Tsianou, P. Alexandridis, Adv. Coll. Interf. Sci. 2020, 275, 102061, https://doi.org/10.1016/j.cis.2019.102061.

[6] P. Singh, Y. Patil, V. Rale, J. Appl. Microbiol. 2019, 126, 2 , https://doi.org/10.1111/jam.14057.

[7] C. Bettenhausen, $C \& E N 2020,98(23)$.

[8] E. Z. Ron, E. Rosenberg, Environ. Microbiol. 2001, 3, 229, https://doi.org/10.1046/j.1462-2920.2001.00190.x.

[9] K. K. Yang, Z. Wu, F. H. Arnold, Nat. Meth. 2019, 16, 687, https://doi.org/10.1038/s41592-019-0496-6.

[10] F. A. Ran, D. Hsu, J. Wright, V. Agarwala, D. A. Scott, F. Zhang, Nat. Protoc. 2013, 8, 2281, https://doi.org/10.1038/nprot.2013.143.

[11] R. D. Patria, J. W. C. Wong, D. Johnravindar, K. Uisan, R. Kumar, G. Kaur, Sustain. Chem. 2021, 2, 237, https://doi.org/10.3390/suschem2020014.

[12] M. Henkel, M. M. Müller, J. H. Kügler, R. B. Lovaglio, J. Contiero, C. Syldatk, R. Hausmann, Proc. Biochem. 2012, 47, 1207, https://doi.org/10.1016/j.procbio.2012.04.018.

[13] S. Rebello, A.N. Anoopkumar, R. Sindhu, P. Binod, A. Pandey, E.M. Aneesh, in "Refining Biomass Residues for Sustainable Energy and Bioproducts", Eds. R.P. Kumar, E. Gnansounou, J.K. Raman, G. Baskar, Elsevier, 2020, pp. 511-521.

[14] A. Moldes, X. Vecino, L. Rodríguez-López, M. Rincón-Fontán, J.M. Cruz, in 'New and Future Developments in Microbial Biotechnology and Bioengineering', Eds. A.G. Rodrigues, Elsevier, 2020, pp. 163-185.

\section{License and Terms}

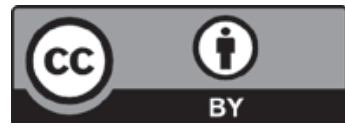

This is an Open Access article under the terms of the Creative Commons Attribution License CC BY 4.0. The material may not be used for commercial purposes.

The license is subject to the CHIMIA terms and conditions: (http:// chimia.ch/component/sppagebuilder/?view=page \&id=12).

The definitive version of this article is the electronic one that can be found at https://doi.org/10.2533/chimia.2021.752 